Revue internationale P.M.E.

Économie et gestion de la petite et moyenne entreprise

\title{
GRH et Genre. Les défis de l'égalité hommes-femmes Annie Cornet, Jacqueline Laufer et Sophia Belghiti-Mahut (dir.) Paris, Vuilbert, coll. " AGRH », 2008
}

\section{Valérie Ballereau}

Volume 22, numéro 1, 2009

URI : https://id.erudit.org/iderudit/038614ar

DOI : https://doi.org/10.7202/038614ar

Aller au sommaire du numéro

Éditeur(s)

Presses de l'Université du Québec

ISSN

0776-5436 (imprimé)

1918-9699 (numérique)

Découvrir la revue

Citer ce compte rendu

Ballereau, V. (2009). Compte rendu de [GRH et Genre. Les défis de l'égalité hommes-femmes Annie Cornet, Jacqueline Laufer et Sophia Belghiti-Mahut (dir.) Paris, Vuibert, coll. « AGRH », 2008]. Revue internationale P.M.E., 22(1), 193-196. https://doi.org/10.7202/038614ar d'utilisation que vous pouvez consulter en ligne. 


\section{Comptes rendus}

GRH ET GENRE.

LES DÉFIS DE L'ÉGALITÉ HOMMES-FEMMES

Annie Cornet, Jacqueline Laufer

et Sophia Belghiti-Mahut (dir.)

Paris, Vuibert, coll. «AGRH», 2008

Cet ouvrage collectif dirigé par Annie Cornet, Jacqueline Laufer et Sophia Belghiti-Mahut apporte un éclairage multidimensionnel sur l'analyse en termes de genre et de différences entre les sexes des questions actuelles relatives à la gestion des ressources humaines dans les entreprises. Les auteures ont structuré leur ouvrage autour de thèmes actuels de la GRH. Chaque thème fait l'objet d'un article particulier qui aborde des problématiques de management à travers le prisme du genre et de la différence entre les sexes. Force est de constater qu'après la lecture de chacun des articles présentés dans cet ouvrage, l'objectif des auteures qui est de «montrer la pertinence (ou l'impertinence!) théorique et pratique d'un regard en termes de genre et de différences entre les sexes» (p. 4) est atteint.

Tout à fait actuel et original dans son contenu, ce livre permet au lecteur d'obtenir un meilleur éclairage sur des problématiques intégrant la notion de genre. Il permet de dépasser les débats quasi idéologiques sur ces sujets et de découvrir des résultats issus de l'observation et de l'analyse de pratiques et de situations d'entreprise. De plus, les études empiriques menées par les chercheurs sont souvent des analyses croisées entre trois pays francophones (la France, la Belgique et le Québec), ce qui accroît l'intérêt de l'ouvrage en offrant un point de vue différent de la vision anglo-saxonne sur ces questions. Un autre intérêt de cet ouvrage collectif réside dans le fait qu'il réunit des résultats de recherche en gestion, mais aussi des recherches menées en sociologie, en psychologie sociale et en psychologie du travail. Le lecteur est invité à parcourir ce livre comme une promenade, en choisissant les articles en fonction de ses intérêts, de ses sensibilités et de ses questionnements, mais aussi avec l'éclairage de ses propres expériences professionnelles.

Dans le premier chapitre rédigé par Claire Gavray, un travail d'analyses statistiques comparées entre la France, le Canada et la Belgique très complet de l'auteure permet de comprendre en quoi le genre est un facteur déterminant dans la compréhension du marché du travail actuel. Ce chapitre est essentiel pour poser l'image chiffrée des différences entre les sexes, d'une part, et cette même image croisée entre les trois pays, d'autre part. 
Le deuxième chapitre interpelle tout particulièrement le lecteur. Jacqueline Laufer décompose très clairement la notion d'égalité professionnelle. Son analyse met en évidence la réalité législative et normative relative à la lutte contre les inégalités de traitement constatées en entreprise, mais ne cache pas la difficulté de prise en compte et d'application par et dans ces mêmes entreprises.

Sabine De Bosscher, Pascale Desrumaux et Annick Durand-Delvigne développent, dans le troisième chapitre, les différences par sexe dans le recrutement. Les conclusions des chercheurs s'appuient sur des recherches menées à la fois en psychologie sociale, en sociologie et en psychologie du travail. Une mise au point sémantique permet de préciser le positionnement des recherches dans ce domaine. La lecture de cet article amène une mise au point sur les stéréotypes qui sous-tendent les processus de recrutement des hommes et des femmes dans les entreprises. La situation des employées peu qualifiées relativement à la formation est traitée par Françoise Lozier dans le chapitre 4. L'auteure part d'un constat historique: la population des emplois peu qualifiés est passée de «majoritairement ouvrière, masculine et industrielle» à «employée, féminine et tertiaire». Or, dans cette population, une nette distinction doit être faite: des femmes souvent diplômées qui n'ont d'autres choix que ce type de poste pour leur accès au marché du travail, d'une part, et, d'autre part, des femmes ayant quitté le système scolaire très tôt et qui ont besoin d'un emploi, quel qu'il soit. L'enjeu majeur réside donc dans la reconnaissance des compétences nécessaires et à développer pour occuper ces emplois dits peu qualifiés.

Dans le chapitre 5, Anne-Françoise Bender et Frédérique Pigeyre s'intéressent, dans un cadre d'analyse institutionnaliste, à la gestion des carrières des femmes. Certains changements sont en cours dans les entreprises. Néanmoins, la gestion des carrières des femmes reste encore très contrastée. Les femmes sont contraintes dans leur choix de carrière par des exigences tant organisationnelles que familiales. Au chapitre 6, Sophia Belghiti-Mahut et Sophie Landrieux-Kartochian dressent une revue de littérature sur la notion de «plafond de verre». L'égalité de traitement des hommes et des femmes est désormais ancrée dans la loi. Ainsi, la recherche de cette égalité n'est plus un combat, mais devient une problématique de la gestion des ressources humaines des entreprises. L'intérêt managérial est clairement mis en évidence, mais la réalité au sein des entreprises aujourd'hui est bien différente. Le changement est contraint par des habitudes organisationnelles fondées sur des «modèles masculins» de gestion de carrière et de temps professionnel.

L'analyse des relations entre le genre et le leadership menée par Annie Cornet et Stéphanie Bonnivert dans le septième chapitre de l'ouvrage 
souligne la persistance du débat sur le sujet. Les auteures explicitent les éléments de ce débat et permettent une prise de recul qui invite à dépasser les stéréotypes pesant tant sur les hommes que sur les femmes dans le management. Dans le chapitre 8, Séverine Lemière et Rachel Silvera mettent en évidence la complexité et la multiplicité des facteurs qui viennent expliquer les inégalités de salaires toujours importantes entre les hommes et les femmes, notamment en Europe. Ces analyses renforcent les stéréotypes de genre et les auteures proposent de questionner ces problématiques non plus par rapport à l'individu, mais par rapport à l'emploi occupé.

Julie Cloutier s'intéresse, dans le chapitre 9, à l'analyse approfondie du processus de construction de la perception de la justice organisationnelle. Elle y relève l'influence des stéréotypes sexuels rattachés aux types d'emplois occupés et l'importance à accorder à des facteurs explicatifs structurels. Nous retiendrons ses conclusions sur une conception commune (homme et femme) de la justice distributive qui montrent qu'adopter des stratégies de rémunération différentes hommes-femmes par les entreprises serait une erreur. Dans le chapitre 10, Marc Dumas aborde la thématique de la conciliation vie familiale-vie professionnelle. Cette problématique de conciliation peut être vue sous deux angles: celui du conflit des temps de vie, mais aussi celui de l'enrichissement de l'un par rapport à l'autre. L'interférence entre l'un et l'autre est réciproque, mais le conflit du travail sur la famille est trois fois plus fréquent que l'inverse. Néanmoins, cette conciliation reste une contrainte forte en premier lieu pour les femmes, puisque les difficultés de conciliation vie familiale-vie professionnelle constituent l'une des causes les plus déterminantes du retrait des femmes de l'emploi salarié.

Dans le onzième chapitre, Angelo Soares traite de l'état des connaissances concernant la question du stress au travail. La souffrance au travail peut-elle être comprise et analysée à travers les différences hommes-femmes? Les hommes seraient-ils plus affectés que les femmes? En l'état actuel de la littérature et des études, aucune conclusion ne peut être apportée. Plutôt que de chercher à comprendre le stress à travers le genre, il semble plus pertinent d'approfondir les recherches relativement à des groupes types d'emplois, de responsabilités et de postes. Enfin, le dernier chapitre de l'ouvrage fait le point sur la place du genre dans la négociation collective. Dans leur travail de recensement, les auteures, Claudette Ross et Julie Cloutier, sont contraintes à relativiser la portée de leurs conclusions en raison du nombre trop restreint d'études empiriques et longitudinales sur les questions soulevées. Leurs conclusions appellent à la poursuite des recherches sur cette thématique.

Il est vrai qu'à la fermeture de l'ouvrage, de nombreuses questions restent sans réponse. Presque tous les auteurs soulignent la pénurie d'études 
empiriques sur les différents domaines traités dans l'ouvrage. En revanche, l'intérêt, la pertinence, l'importance et la nécessité de toutes les questions posées apparaissent comme une évidence. Nous aurions aimé lire une conclusion positive, entraînante, convaincante comme un appel à de futures recherches sur toutes ces thématiques et sur toutes celles qu'elles entraînent.

Nous retiendrons particulièrement ce que plusieurs auteurs ont mis en évidence : la nécessité de dépasser les problématiques relatives aux individus eux-mêmes (hommes-femmes) pour les positionner au plan des emplois et des postes occupés (inégalités de salaires, gestion du stress, formations, justice organisationnelle). Un véritable champ de recherche et d'investigations empiriques vaste et en devenir se dessine, comme l'illustre l'ensemble des articles de cet ouvrage collectif.

Nous recommandons la lecture de ce livre à tous les chercheurs en gestion (et pas seulement en gestion des ressources humaines) afin qu'ils prennent conscience de l'intérêt des questions soulevées. Nous pensons que cette lecture peut susciter des projets de coopération d'équipes de recherche, contribuant à renforcer les réflexions sur les différentes problématiques soulevées dans une approche par le genre. Cet ouvrage s'adresse également aux enseignants en management et en gestion qui trouveront là une occasion d'appréhender avec leurs étudiants (hommes comme femmes) les questions de ressources humaines à travers un prisme différent et jusqu'à aujourd'hui peu traité. Chaque article leur permettra d'approfondir un des principaux thèmes de la gestion des ressources humaines sous ce nouvel angle.

L'ouverture de réflexions autour du genre dès la formation permettra aux futurs salariés de devenir véritablement acteurs de la prise en compte du genre dans le management au quotidien. Enfin, cet ouvrage devrait également être lu avec intérêt par les responsables des ressources humaines et les managers en activité. Les résultats développés dans chacun des chapitres permettent une réelle prise de conscience de la réalité des différences hommesfemmes et de l'état des recherches dans le domaine. La collaboration entre chercheurs et hommes et femmes de terrain est une condition nécessaire à la conduite de recherches empiriques pertinentes.

En conclusion, nous souhaitons que ce livre ouvre des voies de réflexions et mobilise encore davantage d'équipes de recherche autour de ces thématiques modernes.

Valérie Ballereau

Groupe ESC Dijon Bourgogne 